\title{
Violence exposure, affective style, and stress-induced changes in resting state functional connectivity
}

\author{
Heather E. Dark ${ }^{1} \cdot$ Nathaniel G. Harnett ${ }^{1}$ - Adam M. Goodman ${ }^{1}$ - Muriah D. Wheelock ${ }^{1}$ - Sylvie Mrug ${ }^{1}$ • \\ Mark A. Schuster ${ }^{2} \cdot$ Marc N. Elliott $^{3} \cdot$ Susan Tortolero Emery ${ }^{4} \cdot$ David C. Knight $^{1}$
}

Accepted: 13 September 2020 / Published online: 30 September 2020

(C) The Psychonomic Society, Inc. 2020

\begin{abstract}
Chronic childhood stress is linked to greater susceptibility to internalizing disorders in adulthood. Specifically, chronic stress leads to changes in brain connectivity patterns, and, in turn, affects psychological functioning. Violence exposure, a chronic stressor, increases stress reactivity and disrupts emotion regulation processes. However, it is unclear to what extent violence exposure affects the neural circuitry underlying emotion regulation. Individual differences in affective style also moderate the impact of stress on psychological function and can thus alter the relationship between violence exposure and brain function. Resting-state functional connectivity ( $\mathrm{rsFC}$ ) is an index of intrinsic brain activity. Stress-induced changes in rsFC between the amygdala, hippocampus, and prefrontal cortex ( $\mathrm{PFC}$ ) are associated with emotion dysregulation and may elucidate how affective style modulates the relationship between violence exposure and brain connectivity. Therefore, the present study examined the impact of violence exposure and affective style on stress-induced changes in rsFC. Participants $(n=233)$ completed two 6-minute resting-state functional magnetic resonance imaging scans, one before (pre-stress) and one after (post-stress) a psychosocial stress task. The bilateral amygdala, hippocampus, and ventromedial prefrontal cortex (vmPFC) were used as seed regions for rsFC analyses. Significant stress-induced changes in the prefrontal, fronto-limbic, and parieto-limbic rsFC were observed. Further, prestress to post-stress differences in rsFC varied with violence exposure and affective style. These findings suggest that prefrontal, fronto-limbic, and parieto-limbic connectivity is associated with the emotional response to stress and provide new insight into the neural mechanisms through which affective style moderates the impact violence exposure has on the brain.
\end{abstract}

Keywords Violence exposure $\cdot$ Affect $\cdot$ Resting-state $\cdot$ Amygdala $\cdot$ Hippocampus $\cdot$ Ventromedial prefrontal cortex

Childhood violence exposure can have deleterious effects on adolescent and adult psychological functioning (Hanson et al., 2008; Hart \& Rubia, 2012; Mead, Beauchaine, \& Shannon, 2010; Moffitt, 2013; Mrug, Loosier, \& Windle, 2008; Mrug \&

Electronic supplementary material The online version of this article (https://doi.org/10.3758/s13415-020-00833-1) contains supplementary material, which is available to authorized users.

David C. Knight

knightdc@uab.edu

1 Department of Psychology, The University of Alabama at Birmingham, CIRC 235H, 1720 2nd Ave S., Birmingham, AL 35294, USA

2 Kaiser Permanente Bernard J. Tyson School of Medicine, Pasadena, CA, USA

3 RAND Corporation, Santa Monica, CA, USA

4 School of Public Health, The University of Texas Health Science Center at Houston, Houston, TX, USA
Windle, 2010). The adverse impact violence exposure has on psychological functioning may be mediated, in part, by stressinduced changes in brain function (Mead et al., 2010; Moffitt, 2013; Thomason \& Marusak, 2017; Thomason et al., 2015; Weissman et al., 2019; Weissman et al., 2020). Specifically, the interconnections among regions like the amygdala, hippocampus, and ventromedial, dorsomedial, and dorsolateral prefrontal cortex (PFC) mediate emotion expression and regulation processes that may be disrupted in adulthood by stressrelated processes associated with maltreatment and/or violence exposure during childhood (Hart \& Rubia, 2012; Mead et al., 2010; Moffitt, 2013; Thomason \& Marusak, 2017). Disruptions between the interconnections among these brain regions may disrupt otherwise healthy stress responses (Arnsten, 2009; Lupien, McEwen, Gunnar, \& Heim, 2009; Thomason \& Marusak, 2017), which may ultimately lead to greater internalizing (e.g., depression and anxiety) symptomology (Burghy et al., 2012; Herringa et al., 2013; Thomason et al., 2015). Therefore, determining the impact 
childhood exposure to violence has on stress-induced changes in the functional connectivity of the amygdala, hippocampus, and PFC in adulthood may offer novel insight into neural processes that promote emotional dysfunction.

More than $50 \%$ of children in the United States are exposed to violence (i.e., as victims and witnesses) before 17 years of age (Finkelhor, Turner Shattuck \& Hamby, 2015; Mrug et al., 2008), and prior work from cross-sectional studies indicate that this violence rarely occurs in isolation (Finkelhor, Ormrod, \& Turner, 2007, 2009). Instead, violence is often experienced in multiple contexts (e.g., family, school, community) of a child's life (Finkelhor et al., 2007, 2009; Mrug et al., 2008; Mrug \& Windle, 2010; Turner, Shattuck, Finkelhor, \& Hamby, 2016) and includes witnessing and victimization in the form of threats and direct physical violence (Finkelhor et al., 2015; Mrug et al., 2008; Mrug \& Windle, 2010). Thus, violence exposure typically reflects intentional, interpersonal violence rather than unintentional acts (e.g., car accident, natural disaster; Forbes et al., 2012; Moffitt, 2013). Prior work suggests intentional acts of violence are stronger predictors of negative mental health outcomes (Forbes et al., 2012; Jiang, Webster, Robinson, Kassam-Adams, \& Richmond, 2018). In addition, children exposed to a violent incident are more likely to be repeatedly exposed to violence throughout their childhood and adolescence (Finkelhor et al., 2007, 2009; Turner, Finkelhor, \& Ormrod, 2010). Findings from cross-sectional and longitudinal studies suggest that repeated exposure to violence across multiple contexts deprives children of a safe environment in which to cope with the violence they experience (Finkelhor et al., 2007; Hooven, Nurius, Logan-Greene, \& Thompson, 2012). Thus, violence often becomes a persistent condition throughout childhood and adolescence (Finkelhor et al., 2007; Hooven et al., 2012; Mrug et al., 2008; Schwab-Stone et al., 1995), rather than an isolated traumatic event (i.e., car accident, natural disaster). The intentional and interpersonal nature of childhood violence exposure, which often occurs in multiple contexts, may enhance the detrimental outcomes of violence, compared with other types of childhood trauma. Thus, childhood violence exposure appears to be a chronic environmental stressor, which may have unique implications for adolescents as they emerge into adulthood (Finkelhor et al., 2007; Hooven et al., 2012).

Children and adolescents exposed to maltreatment and violence demonstrate greater emotional arousal and hypervigilance, altering their emotional response to acute stressors (De Bellis et al., 1999; Margolin \& Gordis, 2004; Saltzman, Holden, \& Holahan, 2005). Further, repeated violence exposure during childhood and adolescence has been linked to higher rates of internalizing disorders (e.g., depression and anxiety) during childhood, adolescence, and adulthood (Hanson et al., 2008; Hooven et al., 2012; Mrug \& Windle, 2010). Thus, violence exposure during these important developmental periods appears to disrupt the neural systems that underlie the stress response (Admon et al., 2009; Dunn et al., 2017; Mead et al., 2010).

Prospective studies demonstrate that childhood and adolescent maltreatment (e.g., violence exposure) is linked to both structural and functional changes in the brain regions (e.g., PFC, amygdala, hippocampus) that underlie stress reactivity and internalizing symptomology (Butler, Yang, Laube, Kühn, \& Immordino-Yang, 2018; Lambert et al., 2017; Saxbe et al., 2018; Weissman et al., 2020). More specifically, high levels of childhood and adolescent maltreatment and violence exposure are associated with decreased PFC, amygdala, and hippocampal volumes in both adolescence and young adulthood (Dannlowski et al., 2012; Hart \& Rubia, 2012; Lambert et al., 2017; Saxbe et al., 2018). In turn, cross-sectional research has found smaller amygdala and hippocampal volumes in adolescents and adults with internalizing disorders (e.g., depression, posttraumatic stress disorder [PTSD], anxiety; Gilbertson et al., 2002; Morey et al., 2012; Mueller et al., 2013; Redlich et al., 2018), suggesting that changes in these brain regions are associated with internalizing symptoms. Several studies also link childhood maltreatment, violence exposure, and adversity to changes in the brain function of adolescents and adults (Harnett et al., 2019; Herringa et al., 2013; Lambert et al., 2017; Thomason \& Marusak, 2017; van der Werff et al., 2013; Weissman et al., 2019; Yamamoto et al., 2017). For instance, both prospective and retrospective studies demonstrate that adolescents and adults that experienced high levels of childhood maltreatment showed greater amygdala activity to threatening stimuli compared to those with lower levels of childhood maltreatment (Dannlowski et al., 2012; Gerin et al., 2019; Redlich et al., 2018; van Harmelen et al., 2013). Interestingly, amygdala activity appears to mediate the relationship between childhood maltreatment and internalizing symptomology in adolescents and adults (Bremner et al., 2005; Burghy et al., 2012; Gerin et al., 2019; Redlich et al., 2018; van den Bulk et al., 2016). This line of research suggests that childhood maltreatment (e.g., violence exposure) may alter amygdala function and modify the future expression of psychiatric symptoms (Gerin et al., 2019; van den Bulk et al., 2016). Finally, threat-elicited amygdala activity varies with PTSD symptom severity in adults (Bremner et al., 2005; Protopopescu et al., 2005; Shin, Rauch, \& Pitman, 2006; White, Costanzo, Blair, \& Roy, 2015), suggesting the amygdala may be hyperresponsive to emotionally salient stimuli in adults with PTSD (Bremner et al., 2005; Protopopescu et al., 2005; Shin et al., 2006; White et al., 2015). Taken together, prior research suggests that early life stress (e.g., violence exposure, maltreatment, adversity) alters brain function and may impact the expression of psychiatric symptoms in adulthood.

Both early life stress (e.g., violence exposure) and acute stress can influence functional brain connectivity in 
adolescents and adults (Herringa et al., 2013; Neumeister et al., 2018; Saxbe et al., 2018; Thomason \& Marusak, 2017; Thomason et al., 2015; van der Werff et al., 2013; Veer, Oei, Spinhoven, van Buchem, Elzinga, \& Rombouts, 2011; Wang et al., 2014). For instance, both prospective and retrospective studies demonstrate that childhood maltreatment is associated with decreased amygdala-ventromedial PFC (vmPFC) resting state functional connectivity (rsFC) in adolescence (Burghy et al., 2012; Herringa et al., 2013; van der Werff et al., 2013). These types of changes in amygdalavmPFC connectivity may interrupt inhibitory control of the PFC over the amygdala and interfere with the healthy regulation of the emotional response to stress (Burghy et al., 2012; Herringa et al., 2013; van der Werff et al., 2013). The amygdala responds to salient emotional information, and projects to the vmPFC, where the vmPFC appears to evaluate the information and regulate amygdala activity (Delgado, Nearing, LeDoux, \& Phelps, 2008; Johnstone, van Reekum, Urry, Kalin, \& Davidson, 2007; Motzkin, Philippi, Wolf, Baskaya, \& Koenigs, 2015; Phelps, Delgado, Nearing, \& LeDoux, 2004; Quirk \& Beer, 2006; Rauch, Delgado, Nearing, \& LeDoux, 2006). In turn, the amygdala controls the peripheral expression of emotion (Cheng, Knight, Smith, \& Helmstetter, 2006; Cheng, Knight, Smith, Stein, \& Helmstetter, 2003; Knight, Nguyen, \& Bandettini, 2005; Wood, Ver Hoef, \& Knight, 2014). The vmPFC also receives projections from the dorsomedial prefrontal cortex (dmPFC) and dorsolateral prefrontal cortex (dlPFC), which direct attentional resources toward stressors (Delgado et al., 2008; Hare, Camerer, \& Rangel, 2009; Morawetz, Bode, Baudewig, \& Heekeren, 2017; Ochsner, Silvers, \& Buhle, 2012; Roy, Shohamy, \& Wager, 2012). Childhood maltreatment has also been linked to decreased amygdala-insula rsFC during adulthood (van der Werff et al., 2013). The connectivity of the amygdala and insula appears to support the identification of salient emotion-related information (Fan et al., 2015; Menon, 2015) important for responding to environmental stressors. Taken together, this prior work suggests that childhood violence exposure may disrupt the function of these brain regions in adolescence and adulthood (Herringa et al., 2013; Thomason et al., 2015; van der Werff et al., 2013). The dysfunction of these brain regions may, in turn, disrupt acute stress-related emotional processes. However, prior work has not prospectively assessed childhood violence exposure to determine its impact on acute stress-induced changes in rsFC.

The amygdala, hippocampus, and PFC process salient emotional stimuli and regulate emotional expression via projections to the autonomic nervous system and hypothalamicpituitary-adrenal (HPA) axis (Arnsten, 2009; Diorio, Viau, \& Meaney, 1993; Hakamata et al., 2017; Lupien et al., 2009; Vyas, Mitra, Rao, \& Chattarji, 2002). Accordingly, the function of these brain regions underlies important emotion processes that may influence internalizing symptomology
(Burghy et al., 2012; Davidson, 2003; Gerin et al., 2019; Johnstone et al., 2007; Yamamoto et al., 2017). Acute stress appears to alter the function and connectivity of these brain regions. For example, prior work has demonstrated changes in brain function and connectivity to acute laboratory stress (Dedovic et al., 2014; Dedovic et al., 2009; Gilam et al., 2017; Maron-Katz, Vaisvaser, Lin, Hendler, \& Shamir, 2016; Pruessner et al., 2008; Quaedflieg et al., 2015; Wheelock et al., 2016; Wheelock et al., 2018). Acute laboratory stress alters both task-based and resting-state functional connectivity among the dIPFC, dmPFC, vmPFC, ventrolateral PFC (vlPFC), cingulate cortex, insula, inferior parietal lobule (IPL), and the hippocampus in adults (Dedovic et al., 2014; Dedovic et al., 2009; Fan et al., 2015; Gilam et al., 2017; Gold, Morey, \& McCarthy, 2015; Paret et al., 2016; Quaedflieg et al., 2015; Veer et al., 2011; Wheelock et al., 2016; Wheelock et al., 2018). Specifically, stress-elicited activation of the dIPFC, dmPFC, insula, mid cingulate cortex, posterior cingulate cortex (PCC), and parietal lobe, and deactivation of the hippocampus and anterior cingulate cortex (ACC) has been observed during task-based functional magnetic resonance imaging (fMRI) studies (Dedovic et al., 2014; Dedovic et al., 2009; Wheelock et al., 2016). Additionally, amygdala connectivity with the PFC, ACC, PCC, insula, and parietal lobe increases as a function of stress (Fan et al., 2015; Gilam et al., 2017; Gold et al., 2015; Veer et al., 2011). Alterations in the rsFC among these brain regions may in turn enhance stress reactivity by impeding successful communication about stressors. For example, stress-induced changes in rsFC may reflect shifts in the allocation of emotional resources during acute stress (Maron-Katz et al., 2016). As discussed above, childhood violence exposure appears to alter the rsFC of many of these same brain regions (Saxbe et al., 2018; Thomason et al., 2015). Taken together, this prior work suggests that childhood violence exposure may alter the connectivity of brain regions that support important emotion processes (Saxbe et al., 2018). In turn, the altered connectivity of these brain regions may result in the long-term disruption of emotion processes, and ultimately lead to prolonged stress responses in adulthood (Saxbe et al., 2018).

Individual differences in affect (or dispositional mood) can be a protective or risk factor for the negative consequences of stress (Davidson, 2000, 2002, 2003; Hankin \& Abramson, 2001; Karatsoreos \& McEwen, 2011; Meulders, Meulders, \& Vlaeyen, 2014). Affective style reflects individual differences in emotion processes (e.g., emotion reactivity and regulation) that influence emotional experiences (Davidson, 2000; Gross, 1998). For example, positive affect appears to alter the interpretation of stressful events by reframing negative impressions into positive interpretations and is therefore associated with fewer mood disorder symptoms (Harding \& Mezulis, 2017). Hence, a positive affective style may serve as a protective factor, reducing stress reactivity and the later 
development of internalizing disorders (Davidson, 2000; Karatsoreos \& McEwen, 2011; Meulders et al., 2014). In contrast, high levels of stress are linked to greater negative affect and the tendency to attribute negative events to oneself (Hankin \& Abramson, 2001). These findings suggest that negative affect may function as a vulnerability factor through which childhood violence exposure promotes subsequent internalizing symptomology (Davidson, 2000; Karatsoreos \& McEwen, 2011; Meulders et al., 2014). Despite converging evidence, few studies have examined the combined effects of positive and negative affect on the relationship between prior life violence exposure and the acute stress response.

The present study examined the relationship between childhood violence exposure and acute stress-induced changes in $\mathrm{rsFC}$, and whether this relationship is moderated by affective style (i.e., positive and negative affect). We hypothesized that (1) acute changes in amygdala, hippocampus, and vmPFC rsFC, following stress induction, would vary as a function of prior life violence exposure, such that those with greater violence exposure would exhibit greater amygdala and hippocampus rsFC with the PFC, insula, and IPL post-stress, and (2) positive and negative affect would moderate the effect of prior life violence exposure on these stress-induced changes in rsFC. For instance, the relationship between violence exposure and pre-stress versus post-stress changes in rsFC may be blunted by high positive affect and enhanced by high negative affect. This study aims to provide novel insight into the mechanisms through which childhood exposure to violence may influence acute stress-induced changes in functional brain connectivity, and how this relationship is moderated by affective style. Understanding the relationship between childhood violence exposure and acute stress-induced changes in rsFC may provide new knowledge about how childhood violence exposure influences stress reactivity in adulthood.

\section{Method}

\section{Participants}

Two hundred eighty-two participants volunteered for the present study. Forty-nine participants were excluded due to excessive motion, poor data quality, or incomplete data (e.g., not completing both resting state scans); therefore, data for two hundred thirty-three emerging adults from the Birmingham site of the Healthy Passages Study were included in the present data analyses (see Table 1). The Healthy Passages Study was a longitudinal, multisite project designed to identify risk and protective factors for adolescent health (Schuster et al., 2012; Windle et al., 2004) and originally included 1,594 children at the Birmingham site. Participants in the Healthy Passages study were recruited from the 5 th-grade classrooms of public schools. Data were collected at four time points between 2003 and 2017 (see Supplemental Fig. S1). The average age (mean $\pm S D$ ) of the present sample at each time point was $11.24 \pm 0.52$ years at Time $1 ; 13.07 \pm 0.51$ years at Time $2 ; 16.22 \pm 0.54$ years at Time 3 ; and $19.10 \pm 1.14$ years at Time 4. Magnetic resonance imaging (MRI) data were collected (average age: $19.61 \pm 1.20$ years) after the fourth Healthy Passages time point was completed (see Supplemental Fig. S1). There was no difference in the proportion of Black American (BA) and White American (WA) participants, $\chi^{2}(1)=2.08, \mathrm{p}=n s$, in the current sample (BA = 149 , WA $=84$ ) compared with the Healthy Passages sample from Birmingham that did not participate in the present study $(\mathrm{BA}=747, \mathrm{WA}=521)$. However, there was a difference in sex, $\chi^{2}(1)=4.73, p=.032$, with a greater proportion of males in the current sample (female $=101$, male $=132)$ compared with the Birmingham sample that did not participate in the present study (female $=648$, male $=620$ ). There was no difference in violence exposure, $t(1483)=1.17, p=n s$, between the current sample and the Healthy Passages sample that did not participate in the present study. Exclusion criteria for the present study included standard MRI contraindications (e.g., metallic devices, pacemaker, metallic foreign body), left-handedness, previous head injury, loss of consciousness, spinal cord abnormalities, pregnancy, and history of claustrophobia, seizures, psychotic symptoms, and blood or circulation disorders (e.g., sickle cell, anemia, diabetes).

\section{Procedure}

Upon arrival to the laboratory, participants provided written informed consent as approved by the University of Alabama at Birmingham Institutional Review Board. The original Healthy Passages study, from which the participants in the present study were recruited, was approved by the Centers for Disease Control and Prevention and the original study site institutions. Participants completed questionnaires, and two 6minute resting state-fMRI scans, during which they were instructed to remain still with their eyes open and not think about anything in particular. Resting state scans were completed prior to (pre-stress) and after (post-stress) a modified version of the Montreal Imaging Stress Task (MIST; Dedovic et al., 2005). The MIST is a psychosocial stress protocol designed for functional brain imaging settings and consists of computerized mental arithmetic challenges and social evaluative threat. Participants completed two MIST scans (i.e., a Control scan followed by a Stress scan). The MIST conditions were presented in a fixed order to best address the questions of interest in this project by reducing variability related to counterbalancing conditions and carryover effects that develop when the Stress condition precedes the Control condition (Wheelock et al., 2016). The version of the MIST used for the present study has been described in prior work (Goodman et al., 2016; Wheelock et al., 2016). 
Table 1 Participant demographics and descriptive statistics

\begin{tabular}{lllll}
\hline & \multicolumn{1}{l}{ Mean $(S D)$} & & & \\
\cline { 2 - 5 } & Overall $(n=233)$ & Class 1 $(n=158)$ & $20.03(1.41)$ & Class 3 $(n=36)$ \\
\hline Age & $19.61(1.20)$ & $19.41(1.10)$ & $39)$ & $20.00(1.22)$ \\
Race & $149 \mathrm{BA} / 84 \mathrm{WA}$ & $87 \mathrm{BA} / 71 \mathrm{WA}$ & $30 \mathrm{M} / 9 \mathrm{~F}$ & $30 \mathrm{BA} / 6 \mathrm{WA}$ \\
Sex & $132 \mathrm{M} / 101 \mathrm{~F}$ & $80 \mathrm{M} / 78 \mathrm{~F}$ & $22 \mathrm{M} / 14 \mathrm{~F}$ \\
Violence exposure & $3.54(2.73)$ & $2.00(1.29)$ & $6.56(1.34)$ & $7.01(2.62)$ \\
PA & $15.40(5.09)$ & $36.28(7.34)$ & $38.33(7.54)$ & $40.19(8.12)$ \\
NA & $37.23(7.61)$ & $15.18(5.18)$ & $17.28(5.41)$ & $14.36(3.79)$ \\
\hline
\end{tabular}

Note. Data presented as mean (standard deviation); $M=$ male; $F=$ female; $\mathrm{BA}=$ Black American; WA = White American; $N=233$

\section{Measures}

Violence exposure Violence exposure was assessed using the Healthy Passages Violence Exposure measure (Eaton et al., 2006; Mrug et al., 2008; Windle et al., 2004) at each of the four time points described above. Participants reported whether they witnessed (1) a threat of physical violence, (2) actual physical violence, and (3) a threat or actual violence involving a weapon; and whether they were $a$ victim of (1) a threat of physical violence, (2) actual physical violence, (3) a threat or actual violence involving a weapon, and (4) physical violence inflicting an injury that required medical care in the past 12 months. Participants responded to each item using a 4-point scale ranging from 0 (never) to 3 (many times). Internal consistency for the Healthy Passages Violence Exposure measure (Eaton et al., 2006; Mrug et al., 2008; Windle et al., 2004) at each wave was: Wave $1=.748$, Wave $2=.646$, Wave $3=.705$, Wave $4=.705$. Responses to each item on the scale were averaged, and the scale was then summed across all time points to create a composite index of violence exposure (Mrug et al., 2008). Violence exposure was mean centered prior to all analyses. A latent class analysis was also completed using Mplus statistical software to outline patterns of violence exposure across all four time points. The results yielded a three-class solution (see Supplemental Fig. S2). The three-class solution was then used in voxel-wise analyses (described below) to determine the effects of violence exposure trajectories on functional brain connectivity.

Positive and Negative Affect Schedule (PANAS) The PANAS (Watson, Clark, \& Tellegen, 1988) is a self-report measure that assesses trait-positive and trait-negative affect. Participants rated to what extent they felt each of 10 positive and 10 negative emotions in general using a 5-point Likert scale ranging from 1 (very slightly or not at all) to 5 (extremely) to reflect positive affect (PA) and negative affect (NA). Positive and negative emotions were independently summed to reflect PA and NA, respectively. Cronbach's alpha was .864 for positive affect and .798 for negative affect. Both PA and NA were mean centered prior to all analyses.
Participants completed the PANAS at the MRI session, prior to the MRI scan.

Self-reported stress Self-reported stress was assessed retrospectively, outside the scanner following the completion of the post-stress resting-state fMRI scan. The self-reported stress measure included eight statements for both the Control and Stress conditions of the MIST (see the Supplemental material). Participants rated each item on a 5-point scale ranging from 1 (not at all) to 5 (extremely). The scale included four items that were positively worded (e.g., I felt I had control) and four that were negatively worded (e.g., Ifelt overwhelmed). Participants' responses were summed separately for Stress and Control conditions, with total possible scores ranging from 8 to 40 for each condition (Wheelock et al., 2016; Wheelock et al., 2018). Cronbach's alpha for the self-reported stress measure was .844 (Control MIST) and .852 (Stress MIST). Selfreported stress data for 12 participants were not collected.

Skin conductance level (SCL) SCL data were collected using MR compatible physiological monitoring equipment (Biopac Systems; Goleta, CA). SCL data were sampled at $10 \mathrm{kHz}$ using two disposable radio translucent electrodes, attached to the thenar and hypothenar eminence of the nondominant hand. Data were filtered using a 1-Hz Infinite Impulse Response (IIR) low-pass filter, resampled to $250 \mathrm{~Hz}$, and transformed based on the individual participant resistance level using Acqknowledge 4.1.0 (Bach, Flandin, Friston, \& Dolan, 2009). Separate averages of SCL amplitude were acquired for the pre-stress and post-stress resting-state scans. Data acquisition methods were similar to prior work (Knight \& Wood, 2011; Wheelock et al., 2016). Data from 22 participants were not analyzed due to equipment malfunction or low/unmeasurable skin conductance values.

\section{Functional MRI (fMRI)}

Image acquisition MRI data were obtained using a 3T Siemens Allegra scanner. Standard high-resolution T1 
weighted structural magnetization-prepared rapid gradientecho (MPRAGE) images were collected $(\mathrm{TR}=2,300 \mathrm{~ms}$, $\mathrm{TE}=3.9 \mathrm{~ms}$, flip angle $=12^{\circ}, \mathrm{FOV}=25.6 \mathrm{~cm}$, matrix $=$ $256 \times 256$, slice thickness $=1 \mathrm{~mm}$, gap $=0.5 \mathrm{~mm}$ ) prior to the first resting-state scan to serve as an anatomical reference for the fMRI data. Resting-state blood oxygen leveldependent (BOLD) fMRI was measured with a gradientecho echoplanar pulse sequence in an oblique axial orientation $\left(\mathrm{TR}=2,000 \mathrm{~ms}, \mathrm{TE}=30 \mathrm{~ms}\right.$, flip angle $=70^{\circ}, \mathrm{FOV}=24 \mathrm{~cm}$, matrix $=64 \times 64$, voxel size $=3.75 \times 3.75 \times 4.0 \mathrm{~mm}$, slice thickness $=4 \mathrm{~mm}$, no gap).

Preprocessing Images were preprocessed using the Analysis of Functional NeuroImages (AFNI; Cox, 1996) software package, the FMRIB Software Library (FSL; Smith et al., 2004), and MRIcron (Rorden \& Brett, 2000). Echoplanar data for pre-stress and post-stress scans were reconstructed (using the Dicom to Nifti option in MRIcron) and reregistered to minimize movement artifact and generate motion correction parameters for use as covariates in subsequent analyses (using 3 dvolreg in AFNI). Images were then corrected for slice timing offset with a Fourier transformation (using 3dTshift in AFNI) and spatially smoothed using a 4-mm full-widthat-half-maximum Gaussian filter (using 3dmerge in AFNI). Time-course data for tissue-based regressors, including cerebrospinal fluid (CSF) and white matter (WM), were extracted from the functional data set prior to spatial smoothing (using $3 \mathrm{dSeg}$ in AFNI).

\section{Data analyses}

SCL A paired-samples $t$ test was conducted to determine whether SCL differed pre-stress to post-stress. A linear mixed-effects (LME) model analysis was also conducted to determine whether SCL differed pre-stress to post-stress by violence exposure, PA, and NA; all mean centered. Condition was entered as a within-subjects factor $(1=$ pre-stress and $2=$ post-stress), and violence exposure, PA, and NA were entered as continuous factors. Race/ethnicity and sex were entered as covariates. All two-way, three-way, and four-way interactions among violence exposure, PA, NA, and Condition were tested. Statistical analyses were completed using SPSS Statistics software.

Self-reported stress A paired-samples $t$ test was conducted to determine whether self-reported stress differed between the Control and Stress conditions of the MIST. An LME model analysis was also conducted to determine whether selfreported stress differed between the Control and Stress conditions of the MIST by violence exposure, PA, and NA (all mean centered). Condition was entered as a within-subjects factor $(1=$ Control MIST and $2=$ Stress MIST $)$, and violence exposure, PA, and NA were entered as continuous factors.
Race/ethnicity and sex were entered as covariates. All twoway, three-way, and four-way interactions among violence exposure, PA, NA, and Condition were tested. Statistical analyses were completed using SPSS Statistics software.

\section{FMRI}

First-level analyses Individual subject-level analyses were completed using multiple linear regression (3dDeconvolve in AFNI) to account for variables of no interest, including (1) mean CSF time course, (2) mean WM time course, (3) six motion parameters, (4) six motion derivatives, and (5) 111 band-pass time courses (band-pass filter: $0.01<f>0.1 \mathrm{~Hz}$ ). These variables were regressed out of the gray matter (GM) time course for each participant. Time points where $>3 \%$ of voxels were greater than 5 times the median absolute deviation (e.g., outliers) of the time series were excluded from the individual subject analyses similar to prior work (Wood et al., 2015). Excluded volumes were ignored in subsequent statistical analyses. The mean number of included volumes was 177 for pre-stress scans and 175 volumes for post-stress scans (out of a total of 178 possible volumes). Thus, $1-3$ volumes were excluded ( $1 \%)$, on average, from each scan. Participants with less than $80 \%$ useable TRs were excluded from further analyses $(n=1)$. The functional dataset was then normalized to the Talairach and Tournoux stereotaxic coordinate system (Talairach \& Tournoux, 1988). For each participant, a seed (6-mm sphere) was placed in six regions of interest (ROIs) based on coordinates obtained from the Talairach atlas in AFNI - the amygdala: right ( $x: 23 y:-5 z:-15)$, left ( $x:-23 y:-5$ $z:-15)$; hippocampus: right $(x: 30 y:-24 z:-9)$, left ( $x:-30 y:-24$ $z:-9)$; and vmPFC: right ( $x: 12 y: 49 z: 4)$, left ( $x:-12 y: 49 z$ : 4)-resulting in one average time course for each of the six ROI. Six (pre-stress and post-stress) voxel-wise Pearson correlation analyses were conducted to correlate the time series of each ROI with the time series of all other voxels throughout the whole brain. The Pearson correlation analysis resulted in one pre-stress and one post-stress ROI-whole brain correlation map for each ROI (i.e., bilateral amygdala, hippocampus, and vmPFC). Each Pearson correlation value was then converted to a Fisher's $Z$ value to normalize the distribution for each participant, and each map was resampled to 1-mm isotropic voxels.

Group-level analyses (1) Six paired-sample $t$ tests (left and right $=2$ ) were conducted in AFNI using 3dttest++ for the bilateral amygdala-whole brain, hippocampus-whole brain, and vmPFC-whole brain analyses to examine the difference between pre-stress and post-stress rsFC. To reduce familywise error (FWE), a Monte Carlo simulation was conducted (3dClustSim in AFNI), using an uncorrected significance threshold of $p<.005$, to determine the cluster corrected significance threshold. Smoothness was estimated based on the spherical autocorrelation function parameter (3dFWHMx in AFNI) by averaging participants' residual time series from 
the first level analysis, resulting in a voxel-wise cluster threshold of $636 \mathrm{~mm}^{3}$ ( $p_{\text {corrected }}<.05$ ). (2) An LME model analysis was conducted using 3dLME (Chen, Saad Britton, Pine, \& Cox, 2013) in AFNI, to determine whether bilateral amygdala-whole brain rsFC, hippocampus-whole brain rsFC, and vmPFC-whole brain rsFC differed between prestress and post-stress as a function of violence exposure and as a function of the interaction between violence exposure and both PA and NA. Both PA and NA were included in each seed-whole brain analysis. A full factorial model was conducted examining all main effects and two-way, three-way, and four-way interactions. The ROI-whole brain Fisher's $Z$ maps were used as the dependent variable for each separate LME analysis. Pre-stress and post-stress scans were coded and entered into the model as a repeated, within-subjects factor: Condition $(1=$ pre-stress and $2=$ post-stress $)$. Race and sex were included as covariates in both analyses.

Follow-up analyses After completion of the LME analysis for each ROI, follow-up analyses were conducted to further examine significant interactions. First, for each interaction term, the average Fisher's $Z$ values were obtained for each significant volume of activity for both pre-stress and post-stress scans. If the significant interaction included Condition (e.g., pre-stress to post-stress difference), two separate follow-up analyses were completed, one using pre-stress $\mathrm{rsFC}$, and the other using post-stress rsFC as the dependent variable to determine how the interaction of violence exposure, PA, and NA varied with rsFC pre-stress versus post-stress. For main effects and significant interaction terms that did not include Condition, pre-stress rsFC and post-stress $\mathrm{rsFC}$ data were averaged to reflect an overall rsFC value and used as the dependent variable for follow-up analyses. Using PROCESS (Hayes, 2012; Hayes \& Preacher, 2013), a multiple regression analysis was conducted to compute simple slopes for each significant interaction. Each simple slopes analysis examined the conditional effects of violence exposure on rsFC at different levels of the moderators (e.g., PA, NA): one standard deviation below the mean (low) and one standard deviation above the mean (high) (Hayes, 2012). In addition, partial correlation was used as a follow-up analysis of a significant Condition $\times$ Violence Exposure interaction. The partial correlation analysis compared violence exposure with both prestress and post-stress rsFC, while controlling for PA, NA, race, and sex to determine the relationship between violence exposure and rsFC during pre-stress and post-stress scans.

Violence exposure class and rsFC Differences in pre-stress to post-stress $\mathrm{rsFC}$ among the three violence exposure classes were assessed using AFNI's 3dttest++ with a covariate for violence exposure class (dependent measures: left/right amygdala-whole brain, left/right hippocampus-whole brain, and left/right vmPFC-whole brain). A voxel-wise cluster threshold of $636 \mathrm{~mm}^{3}$ ( $p_{\text {corrected }}<.05$ ) was also applied to this analysis.

Violence exposure class and $\mathrm{SCL}$ A repeated-measures ANOVA (dependent measure: SCL) was conducted using SPSS Statistics software to determine whether there were pre-stress to post-stress differences among the three violence exposure classes.

Violence exposure class and self-reported stress A repeatedmeasures ANOVA (dependent measure: self-reported stress) was conducted using SPSS Statistics software to determine whether there were differences in self-reported stress among the three violence exposure classes.

\section{Results}

Descriptive statistics Descriptive statistics are presented in Table 1.

\section{Behavioral results}

Skin conductance level (SCL) Results from the paired-samples $t$ test demonstrate that SCL was greater during post-stress ( $M$ $=8.61, S E M=0.48)$ than pre-stress $(M=7.64, S E M=0.44)$, $t(210)=5.78, p<.001$, scans. This finding suggests that in general the psychosocial stress task elicited a physiological response. The LME analysis revealed a significant main effect for NA, $F(1,192)=8.98, p=.003$, such that SCL varied positively with NA $(r=.161, p=.020)$. There was also a significant PA $\times \mathrm{NA}$ interaction, $F(1,192)=15.07, p<$ .001. A test of simple slopes was completed to further assess the PA $\times$ NA interaction. It revealed that among those with high NA, SCL varied negatively with PA $(b=-.159, p=$ .024), while there was no relationship between SCL and PA among those who reported low NA $(b=-.108, p=.165)$. There were no other significant effects.

Self-reported stress Results from the paired-samples $t$ test demonstrate that self-reported stress was greater during the Stress $(M=25.79, S E M=0.45)$ than the Control $(M=$ 14.96, $S E M=0.38), t(220)=19.70, p<.001$, condition of the MIST, which suggests that the procedures used in the present study successfully manipulated stress across conditions. The LME analysis revealed a significant main effect for PA, $F(1,202)=7.19, p=.008$, such that self-reported stress varied negatively with PA $(r=-.173, p=.011)$. There was also a significant Condition $\times$ Violence Exposure interaction, $F(1,202)=11.67, p=.001$. Specifically, self-reported stress for the Control MIST varied positively with violence exposure $(r=.165, p=.015)$, while self-reported stress for the 
Stress MIST did not vary with violence exposure $(r=-.027, p$ $=n s)$. Finally, there was a significant Condition $\times \mathrm{PA} \times \mathrm{NA}$ interaction, $F(1,202)=3.90, p=.049$. A test of simple slopes revealed that among those who reported low NA, there was a negative relationship between self-reported stress and PA for the Control MIST ( $b=-.177, p=.011)$, while there was no relationship between PA and self-reported stress for the Stress MIST $(b=.0005, p=n s)$. Among those who reported high NA, there was no relationship between self-reported stress and PA for the Control MIST $(b=-.079, p=n s)$, while selfreported stress varied negatively with PA for the Stress MIST $(b=-.153, p=.05)$. There were no other significant effects.

\section{Resting state functional connectivity (rsFC) results}

Pre- to post-stress differences in amygdala-, hippocampus-, and vmPFC-whole brain rsFC Six paired-samples $t$ tests were conducted to determine whether there were differences in bilateral amygdala, hippocampus, and vmPFC rsFC. Results of these analyses are presented in Supplemental Figs. S3-S5 and Supplemental Tables S1-S3.

Relationship between SCL and rsFC Bivariate correlation analyses were conducted to compare differential (post-stress minus pre-stress) SCL and differential (post-stress minus prestress) rsFC. Differential SCL varied positively with differential left vmPFC-right insula $\operatorname{rsFC}(r=.136, p=.048$, uncorrected). There were no other significant correlations. Bivariate correlation analyses were also conducted to determine whether pre-stress and post-stress SCL varied with prestress and post-stress rsFC, respectively. SCL varied with rsFC among many regions during pre-stress and post-stress scans (see Supplemental Tables S4-S6).

Relationship between self-reported stress and rsFC Bivariate correlation analyses were conducted to compare differential (Stress minus Control MIST) self-reported stress and differential (post-stress minus pre-stress) rsFC. Differential selfreported stress varied positively with differential right amygdala-left dlPFC rsFC ( $r=.143, p=.034$, uncorrected $)$. Further, differential self-reported stress varied with differential right vmPFC-left dlPFC $\operatorname{rsFC}(r=-.132, p=.049$, uncorrected). Differential self-reported stress also varied positively with differential right vmPFC-right STG rsFC (see Supplemental Results). There were no other significant correlations. Bivariate correlation analyses were also conducted to determine whether self-reported stress for the Control and Stress conditions of the MIST varied with pre-stress and post-stress rsFC. Self-reported stress for the Control and Stress conditions of the MIST varied with the pre-stress and post-stress rsFC of several brain regions (see Supplemental Tables S7-S9).

\section{Linear mixed-effects (LME) analysis}

A large number of results were obtained from the LME analysis of the rsFC data. Full results from the rsFC LME analyses are presented in the Supplemental materials (Supplemental Results; Supplemental Tables S10-S15). The LME results presented in the following sections include those that are focused on the primary aims of the present study (i.e., examining the relationships among violence exposure, affective style, and rsFC in regions of interest) and are included in the Discussion.

Amygdala (Condition $\times$ PA $\times$ NA $\times$ Violence Exposure) $\mathrm{A}$ Condition $\times \mathrm{PA} \times \mathrm{NA} \times$ Violence Exposure interaction was observed in the rsFC of the right amygdala with the left IPL (see Supplemental Table S10). Differences in affective style modulated the relationship between violence exposure and right amygdala connectivity with the left IPL pre-stress to post-stress. Specifically, violence exposure varied positively with post-stress, but not pre-stress rsFC among those with low NA and low PA (see Fig. 1a; Supplemental Table S11). Among those with low NA and high PA, violence exposure varied positively with prestress, but not post-stress rsFC (see Fig. 1b; Supplemental Table S11).

Hippocampus (Condition $\times \mathrm{PA} \times \mathrm{NA} \times$ Violence Exposure) $\mathrm{A}$ Condition $\times \mathrm{PA} \times \mathrm{NA} \times$ Violence Exposure interaction was observed in the rsFC of the left hippocampus with the left mid cingulate gyrus and the left dmPFC (see Supplemental Table S12). Differences in affective style modulated the relationship between violence exposure and the rsFC of these regions from pre-stress to post-stress. Specifically, violence exposure varied positively with the rsFC of these regions pre-stress, but not post-stress, among those with low NA and low PA (see Fig. 2a; Supplemental Table S13). Violence exposure also varied positively with left hippocampus-left cingulate rsFC post-stress, but not pre-stress, among those with high NA and low PA (see Supplemental Table S13). Further, violence exposure varied positively with left hippocampus-left dmPFC rsFC post-stress, but not pre-stress, among those with low NA and high PA (see Fig. 2b; Supplemental Table S13).

VmPFC (Condition $\times \mathrm{PA} \times \mathrm{NA} \times$ Violence Exposure) $\mathrm{A}$ Condition $\times \mathrm{PA} \times \mathrm{NA} \times$ Violence Exposure interaction was observed in the rsFC of the right vmPFC to the left dmPFC (see Supplemental Table S14). Differences in affective style modulated the relationship between violence exposure and right vmPFC connectivity with the left dmPFC pre-stress to post-stress. Specifically, violence exposure varied negatively with right vmPFC-left dmPFC rsFC post-stress among those with high NA and high PA (see Fig. 3a; Supplemental Table S15). Violence exposure also 

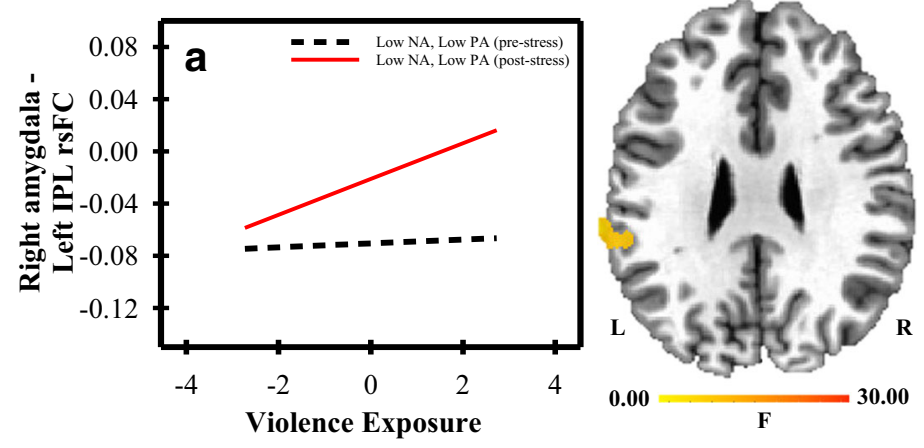

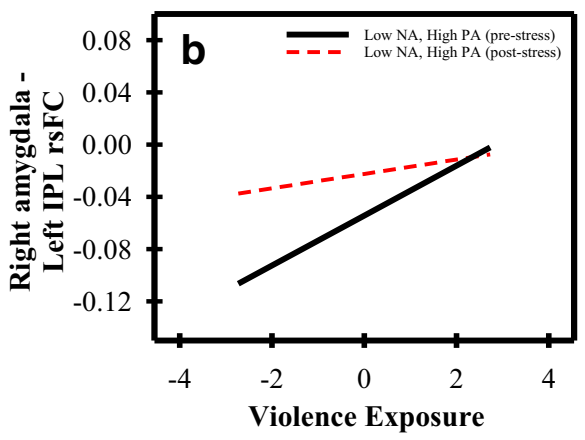

$\mathrm{PA}$, higher violence exposure was associated with greater right amygdala-left IPL rsFC post-stress (red solid line), while no relationship was observed pre-stress (black dashed line). b Among those low NA and high PA, higher violence exposure was associated with greater right amygdala-left IPL rsFC pre-stress (solid black line), while no relationship was observed post-stress (dashed red line). Violence exposure and both PA and NA were mean centered prior to conducting all LME analyses. Cluster threshold $=636 \mathrm{~mm}^{3} ; p_{\text {FWE }}<.05 ; N=233$

Amygdala Left and right amygdala rsFC did not vary prestress to post-stress with violence exposure class.

Hippocampus Left hippocampus-left dlPFC rsFC varied prestress to post-stress with violence exposure class, $t(231)=-$ $3.65, p_{\mathrm{FWE}}=.05$ (see Supplemental Fig. S6). Participants in Class 3 demonstrated greater pre-stress (mean $=.159, S E M=$ .024 ) than post-stress (mean $=.047, S E M=.026)$ rsFC. Further, participants in Class 3 (mean $=.159$, SEM $=.027$ ) demonstrated greater pre-stress rsFC than participants in Class $1($ mean $=.090$, SEM $=.011$; see Supplemental Fig. S6). No pre-stress differences were observed between Classes 1 and 3 with Class 2 (mean $=.124$, SEM $=.024$; see Supplemental Fig. S6). There were no differences in post-stress rsFC. Right hippocampus rsFC did not vary pre-stress to post-stress with violence exposure class.
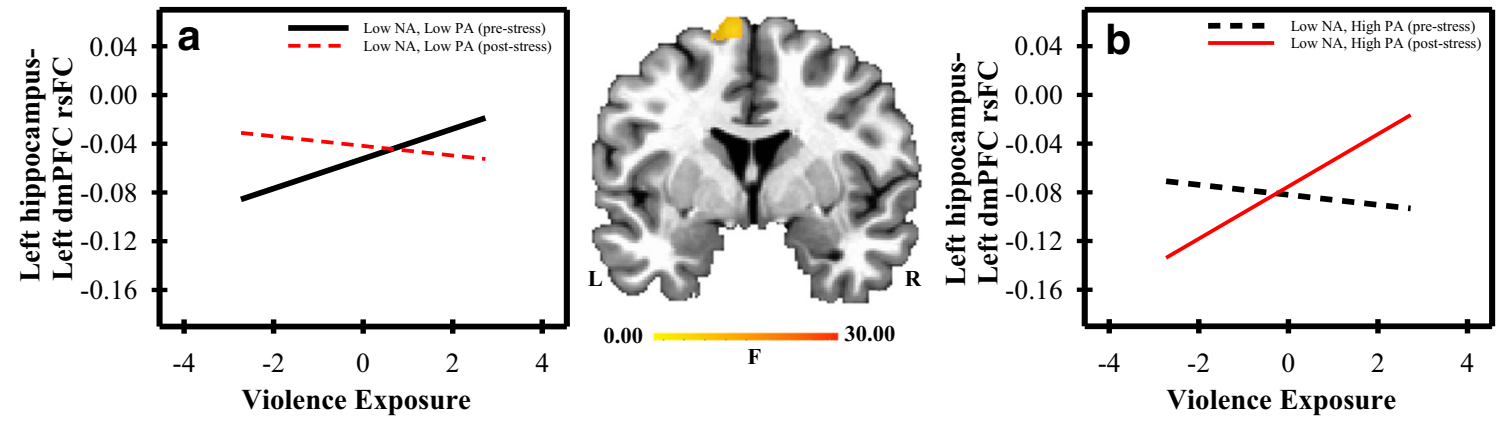

Fig. 2 Left hippocampus-left dorsomedial prefrontal cortex (dmPFC) resting state functional connectivity (rsFC). The figure depicts the significant Condition $\times$ Positive Affect $(\mathrm{PA}) \times$ Negative Affect $(\mathrm{NA}) \times$ Violence Exposure interaction results from the left hippocampus linear mixed-effects (LME) analysis. The graphs show the simple slopes analysis elucidating the relationship between pre-stress and post-stress left hippocampus-left dmPFC rsFC and violence exposure by affective style. Significant slopes are represented by solid lines, and nonsignificant slopes are represented by dashed lines. a Among those with low NA and low
$\mathrm{PA}$, violence exposure varied positively with left hippocampus-left dmPFC rsFC pre-stress (black solid line), while no relationship was observed post-stress (red dashed line). b Among those low NA and high PA, violence exposure varied positively with left hippocampus-left dmPFC rsFC post-stress (solid red line), while no relationship was observed prestress (black dashed line). Violence exposure and both PA and NA were mean centered prior to conducting all LME analyses. Cluster threshold = $636 \mathrm{~mm}^{3} ; p_{\text {FWE }}<.05 ; N=233$ 

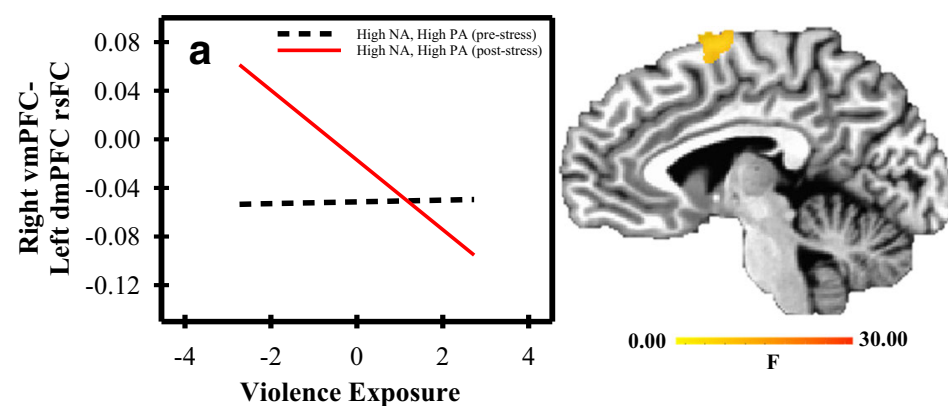

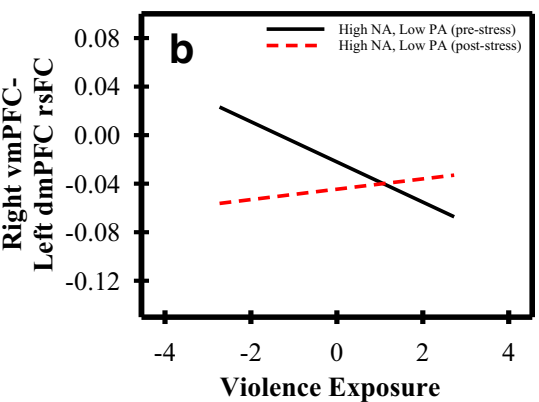

high NA and high PA, greater violence exposure was associated with decreased right vmPFC-left dmPFC rsFC post-stress (red solid line), while no relationship was observed pre-stress (black dashed line). b Among those high NA and low PA, greater violence exposure was associated with decreased right vmPFC-left dmPFC rsFC pre-stress (solid black line), while no relationship was observed post-stress (red dashed line). Violence exposure and both PA and NA were mean centered prior to conducting all LME analyses. Cluster threshold $=636 \mathrm{~mm}^{3} ; p_{\mathrm{FWE}}<$ $.05 ; N=233$
VmPFC The left and right vmPFC did not vary pre-stress to post-stress with violence exposure class among hypothesized regions. However, left vmPFC rsFC varied with the right culmen (cerebellum; see Supplemental Results and Supplemental Fig. S7).

Violence exposure class and SCL SCL did not vary prestress to post-stress with violence exposure classes, $F(1,208)$ $=.602, p=n s$.

Violence exposure class and self-reported stress There was a significant difference in Control versus Stress MIST selfreported stress by violence exposure class, $F(1,218)=$ 5.63, $p=.004$. Participants in Classes 1-3 demonstrated greater self-reported stress to the Stress MIST compared with the Control MIST: Class 1 (Control MIST: mean = 14.63, $S E M=0.460$; Stress MIST: mean $=26.74, S E M=$ 0.546), $t(218)=-18.35, p<.001$; Class 2 (Control MIST: mean $=15.42, S E M=0.905 ;$ Stress MIST: mean $=23.55$, $S E M=1.073), t(218)=-6.26, p<.001$; Class 3: (Control MIST: mean $=15.86, S E M=0.930 ;$ Stress MIST $:$ mean $=$ 24.31, $S E M=1.103), t(218)=-6.33, p<.001$. Further, participants in Class 1 (mean $=26.74, S E M=.546)$ reported higher self-reported stress during the Stress MIST than participants in Class $2($ mean $=23.55, S E M=1.073)$, $t(218)=2.64, p=.009$, and participants in Class 3 (mean $=24.31, S E M=1.103), t(218)=1.97, p=.049$ (see Supplemental Fig. S8).

\section{Discussion}

Repeated exposure to violence during childhood is linked to chronic emotion dysregulation and, in turn, greater susceptibility to internalizing psychopathology (Hanson et al., 2008; Mead et al., 2010; Mrug \& Windle, 2010). Specifically, repeated exposure to violence appears to modify functional connectivity patterns within the brain that control emotion regulation processes (Saxbe et al., 2018; Thomason \& Marusak, 2017; Thomason et al., 2015). Emotion regulation processes rely upon the connectivity of limbic, parietal, and prefrontal brain regions that may underlie internalizing symptomology by disrupting both the interpretation of and response to stressful events (Ochsner et al., 2012; Young \& Koenigs, 2007). Therefore, determining the impact of violence exposure on functional brain connectivity may offer new insight into the neural processes that affect successful emotion regulation among those exposed to violence. Understanding the manner through which affective style modulates the relationship between violence exposure and brain connectivity would provide novel insight into individual differences in the development of emotional dysfunction. The present study examined the impact violence exposure and affective style have on stress-induced changes in functional brain connectivity. We found stress-induced changes in rsFC among prefrontal, fronto-limbic, and parieto-limbic regions that support the expression and regulation of emotion. Further, affective style moderated the relationship between violence exposure and functional brain connectivity. These findings suggest that the functional connectivity of prefrontal, fronto-limbic, and parieto-limbic regions that support emotion processes vary with childhood violence exposure and affective style.

Amygdala rsFC In the present study, we found that amygdalaIPL rsFC varied with violence exposure as a function of affective style (see Fig. 1; Supplemental Tables S10 and S11). 
The amygdala is an important component of the neural circuity that underlies the peripheral expression of emotion (Cheng et al., 2006; Cheng et al., 2003; Klumpers, Kroes, Baas, \& Fernández, 2017; Knight et al., 2005; Orem et al., 2019; Wood et al., 2014), while the IPL is important for the topdown attentional control of emotion (Sylvester et al., 2012). Among participants with low NA and low PA, post-stress amygdala-IPL rsFC varied positively with violence exposure (see Fig. 1a; Supplemental Tables S10 and S11). Prior work indicates that the amygdala and IPL are important for responding to emotion-related threats and coactivate during emotion regulation processes (Alarcón, Sauder, Teoh, Forbes, \& Quevedo, 2019). Further, task-based functional connectivity research has found that amygdala-IPL connectivity increases during psychosocial stress (Fan et al., 2015). Thus, this prior work is generally consistent with the present findings that suggest high violence exposure is associated with greater coupling of the amygdala and IPL in those with both low NA and low PA (see Fig. 1a). The present findings suggest that violence exposure interacts with affect. More specifically, high violence exposure in combination with lower overall NA and PA may be associated with greater processing of emotional information in response to acute stress. In the present study, we hypothesized that the relationship between violence exposure and post-stress rsFC would be enhanced by high NA, and blunted by high PA. Our findings indicate that while high NA had limited impact on the relationship between violence exposure and amygdala-IPL rsFC, high PA diminished the apparent impact violence exposure has on amygdala-IPL rsFC post-stress (see Fig. 1b). This finding is generally consistent with the view that high PA may blunt the impact adolescent violence exposure has on stress-induced changes in amygdala-IPL connectivity.

Hippocampus rsFC The hippocampus is important for the consolidation of emotionally salient memories (Phelps, 2004; Richter-Levin \& Akirav, 2001) and stress-induced hippocampal activity decreases along with the activity of the medial orbitofrontal cortex (OFC) and ACC (Dedovic et al., 2009; Pruessner et al., 2008). Further, prior work shows that adult hippocampus-dmPFC rsFC varies as a function of childhood trauma (Birn, Patriat, Phillips, Germain, \& Herringa, 2014). The present study advances this prior work, showing that hippocampus-dmPFC rsFC varied pre-stress to poststress as a function of affective style and violence exposure. More specifically, post-stress hippocampus-dmPFC rsFC increased as violence exposure increased among those with low NA and high PA (see Fig. 2; Supplemental Tables S12 and $\mathrm{S} 13)$. We hypothesized that the relationship between violence exposure and post-stress rsFC would be blunted by high PA. Instead, we only found a relationship between violence exposure and the rsFC of these regions when PA was high. Thus, PA did not attenuate the impact of violence exposure, and actually enhanced the effects on the rsFC of the hippocampus and dmPFC. Although the current findings do not precisely match our a priori hypothesis, they do suggest that PA may influence the connectivity and communication between the hippocampus and dmPFC. The dmPFC is important for the appraisal of threatening stimuli, while the hippocampus supports memory retrieval processes during acute stress and modulates HPA axis activity (Goodman, Harnett, \& Knight, 2018; Kalisch, Wiech, Critchley, \& Dolan, 2006; Kim \& Diamond, 2002). Taken together with this prior work, the present findings suggest that among those with high PA and low NA, increased hippocampal-dmPFC rsFC may reflect differences in the appraisal of psychosocial stress and subsequent modulation of the stress response among those exposed to varying levels of violence.

VmPFC rsFC The vmPFC is important for generating affective meaning as well as regulating behavioral and physiological responses (Roy et al., 2012). Further, connectivity between distinct regions of the PFC are important for emotion regulation (Hare et al., 2009; Ochsner et al., 2012). In the present study, vmPFC-dmPFC rsFC varied pre-stress to post-stress as a function of violence exposure and affective style. Specifically, among those with both high NA and PA, greater violence exposure was negatively associated with post-stress vmPFC-dmPFC rsFC (see Fig. 3; Supplemental Tables S14 and S15). These findings suggest that those with higher NA and $\mathrm{PA}$ and lower violence exposure, have greater functional coupling of brain regions (i.e., vmPFC and dmPFC) that are important for the regulation of emotion in response to psychosocial stress. Our findings, in combination with prior work (Kalisch et al., 2006; Morawetz et al., 2017; Phan et al., 2005), suggest that those with higher levels of NA and PA may be more attentive to emotional stimuli. Further, their ability to regulate the emotional response to psychosocial stress may vary as a function of violence exposure. Taken together, these findings suggest that individual differences in emotion regulation may explain variability in stress reactivity among young adults exposed to childhood violence.

SCL, self-reported stress, and rsFC Secondary analyses were completed to determine whether SCL and self-reported stress were associated with rsFC. We found that differential rsFC between the vmPFC and insula varied positively with differential SCL. This finding suggests the functional coupling of these brain regions may underlie stress-related changes in autonomic activity. The vmPFC is important for assigning value to stimuli, guiding adaptive behavior, and regulating the emotional response, while the insula is important for interoceptive awareness (Damasio, 1994; Hare et al., 2009; Hiser \& Koenigs, 2018; Sinha, Lacadie, Constable, \& Seo, 2016). Prior research indicates that stress-related tasks activate the vmPFC and insula (Sinha et al., 2016), and that activity within 
these brain regions varies with skin conductance responses (Nagai et al., 2004). Therefore, the increased stress-induced rsFC observed among these brain regions in the present study provides support for the view that vmPFC and insula connectivity plays an important role in the modulation of the peripheral emotional response (Nagai et al., 2004; Thayer \& Lane, 2000). In addition, we found that differential rsFC between the amygdala and dIPFC varied positively with differential selfreported stress. While prior work indicates the amygdala mediates the peripheral emotional response (Cheng et al., 2006; Cheng et al., 2003; Knight et al., 2005; Orem et al., 2019; Wood et al., 2014), the present study advances this prior work by demonstrating that amygdala connectivity with the dIPFC also appears to be important for the subjective experience of stress. The dIPFC supports working memory and attentional processes that play an important role in the top-down control of the amygdala (Comte et al., 2016; Delgado et al., 2008; Ochsner et al., 2009; Ochsner et al., 2012; Sylvester et al., 2012). Therefore, increased rsFC between the amygdala and dIPFC may reflect greater recruitment of these regions in response to acute stress. The findings from these secondary analyses suggest that changes in differential rsFC among these brain regions may reflect greater stress reactivity.

Violence exposure class and rsFC The pre-stress to post-stress rsFC of the left hippocampus and left dIPFC appears to differ among the three violence exposure classes identified in the present study (see Supplemental Figs. S2 and S6). Specifically, we observed greater positive hippocampusdlPFC rsFC among participants in Class 3 (i.e., moderate violence exposure in early adolescence that subsequently increased through late adolescence) compared with participants in Class 1 (i.e., low violence exposure throughout adolescence), which suggests greater baseline coupling of these regions (see Supplemental Fig. S6). Specifically, those who experienced moderate levels of violence that increased throughout adolescence (i.e., Class 3) may exhibit greater changes in the connectivity between the dIPFC and hippocampus in comparison to those who experienced consistently low violence exposure throughout adolescence. These findings suggest that the pattern of violence experienced during adolescence differentially affects the rsFC of the dIPFC and hippocampus. Further, acute stress decreased the positive rsFC among those in Class 3, but not those in Classes 1 or 2 (see Supplemental Fig. S6). This finding suggests that violence exposure that increases throughout adolescence may negatively impact the neural response to stress. The dIPFC is important for top-down emotion processes and modulates hippocampal activity (Benoit \& Anderson, 2012; Benoit, Hulbert, Huddleston, \& Anderson, 2015). Thus, decreased connectivity among these brain regions is consistent with the view that psychosocial stress reduces the dlPFC's inhibitory control over the hippocampus among participants in Class 3. Prior work suggests that childhood violence exposure often becomes a chronic environmental condition rather than a series of isolated incidents (Finkelhor et al., 2007; Hooven et al., 2012; Mrug et al., 2008; Schwab-Stone et al., 1995). Thus, the experience of participants in Class 3 may more closely reflect this chronic environmental condition than the experiences of participants in Class 1, who have experienced consistently low levels of violence, as well as participants in Class 2 , who encountered initial high levels of violence exposure that subsequently decreased across adolescence. The present study suggests that low or decreasing levels of violence during adolescence may have a minimal impact on the connectivity of the hippocampus and dIPFC, regions that underlie important aspects of the stress response (Ochsner et al., 2012; Phelps, 2004; Richter-Levin \& Akirav, 2001). Additionally, Class 3 showed greater pre-stress to post-stress changes in the rsFC of these brain regions. Changes in the connectivity among these regions may negatively impact the stress response and subsequently lead to increased susceptibility to internalizing disorders. Prior work suggests that cumulative violence exposure is more important for predicting internalizing symptomology than the type of violence experienced (Mrug et al., 2008). Results from the present study advance this prior work, suggesting that the trajectory of violence exposure may also impact the connectivity of brain regions that influence internalizing symptoms. The present findings suggest that moderate levels of violence exposure in early adolescence that subsequently increase through late adolescence may result in greater susceptibility to the effects of acute stress. Future studies should consider both the trajectory as well as cumulative violence exposure when examining the impact of violence exposure on negative psychological outcomes.

Limitations The present study focused on emerging adult participants. Emerging adulthood is an important stage of life that is marked by increased risk for psychopathology (Arnett, 2014). However, the functional brain connectivity demonstrated during this period may not yet reflect adult functional brain connectivity, as the brain continues to develop into young adulthood (Taber-Thomas \& Pérez-Edgar, 2015). Specifically, neurodevelopmental changes in fronto-limbic connections as well as cortical structures may not have reached full maturation during emerging adulthood, and thus may not reflect fully developed adult brain function (TaberThomas \& Pérez-Edgar, 2015). Additionally, participants in the present study completed one neuroimaging session. It is possible that preexisting connectivity differences or environmental factors during development contributed to differences in functional connectivity observed in the present study. Future studies should employ longitudinal neuroimaging designs to assess functional changes in the brain over time. Finally, the present study found that the relationship between 
pre-stress to post-stress changes in rsFC and violence exposure varied with affective style. This relationship is consistent with our general hypothesis that affective style modulates stress-elicited changes in rsFC. However, it is also possible that childhood violence exposure modulates the relationship between affective style and rsFC. Additional studies are need to fully disentangle these possible explanations.

Conclusion Violence exposure during childhood and adolescence alters rsFC patterns in emerging adulthood among brain regions involved in emotion expression and regulation. Changes in the rsFC of brain regions that support emotion regulation processes may alter the ability to effectively regulate the emotional response to stress. Stress-induced changes in fronto-limbic, prefrontal, and parieto-limbic rsFC varied with violence exposure, suggesting that exposure to violence alters the functional connectivity of brain regions that support emotion-related processes. Furthermore, the relationship between violence exposure and the functional networks that support emotion regulation was moderated by PA and NA, indicating that individual differences in affective style play a role in how emerging adults exposed to violence during development respond to stress.

Acknowledgements This research was supported by the National Institutes of Health (Grant Number: 1R01 MH098348). Data from the Healthy Passages study was supported by cooperative agreements (CCU409679, CCU609653, CCU915773, U48DP000046, U48DP000057, U48DP000056, U19DP002663, U19DP002664, and U19DP002665) with the Centers for Disease Control and Prevention.

Open practices statement None of the data or materials for the experiments reported here is available, and none of the experiments were preregistered.

\section{References}

Admon, R., Lubin, G., Stern, O., Rosenberg, K., Sela, L., Ben-Ami, H., \& Hendler, T. (2009). Human vulnerability to stress depends on amygdala's predisposition and hippocampal plasticity. Proceedings of the National Academy of Sciences of the United States of America, 106(33), 14120-14125.

Alarcón, G., Sauder, M., Teoh, J. Y., Forbes, E. E., \& Quevedo, K. (2019). Amygdala functional connectivity during self-face processing in depressed adolescents with recent suicide attempt. Journal of the American Academy of Child \& Adolescent Psychiatry, 58(2), 221-231.

Arnett, J. J. (2014). Emerging adulthood: The winding road from the late teens through the twenties. Oxford University Press, USA.

Arnsten, A. F. T. (2009). Stress signalling pathways that impair prefrontal cortex structure and function. Nature Reviews Neuroscience, 10(6), 410-422. https://doi.org/10.1038/nrn2648

Bach, D. R., Flandin, G., Friston, K. J., \& Dolan, R. J. J. (2009). Timeseries analysis for rapid event-related skin conductance responses. Journal of Neuroscience Methods, 184, 224-234. https://doi.org/10. 1016/j.jneumeth.2009.08.005
Benoit, R. G., \& Anderson, M. C. (2012). Opposing mechanisms support the voluntary forgetting of unwanted memories. Neuron, 76(2), 450-460.

Benoit, R. G., Hulbert, J. C., Huddleston, E., \& Anderson, M. C. (2015). Adaptive top-down suppression of hippocampal activity and the purging of intrusive memories from consciousness. Journal of cognitive neuroscience, 27(1), 96-111.

Birn, R. M., Patriat, R., Phillips, M. L., Germain, A., \& Herringa, R. J. (2014). Childhood maltreatment and combat posttraumatic stress differentially predict fear-related fronto-subcortical connectivity. Depression and Anxiety, 31(10), 880-892.

Bremner, J. D., Vermetten, E., Schmahl, C., Vaccarino, V., Vythilingam, M., Afzal, N., ... Charney, D. S. (2005). Positron emission tomographic imaging of neural correlates of a fear acquisition and extinction paradigm in women with childhood sexual-abuse-related posttraumatic stress disorder. Psychological Medicine, 35(6), 791.

Burghy, C. A., Stodola, D. E., Ruttle, P. L., Molloy, E. K., Armstrong, J. M., Oler, J. A., ... Essex, M. J. (2012). Developmental pathways to amygdala-prefrontal function and internalizing symptoms in adolescence. Nature Neuroscience, 15(12), 1736-1741.

Butler, O., Yang, X. F., Laube, C., Kühn, S., \& Immordino-Yang, M. H. (2018). Community violence exposure correlates with smaller gray matter volume and lower IQ in urban adolescents. Human Brain Mapping, 39(5), 2088-2097.

Chen, G., Saad Z. S., Britton, J. C., Pine D. S., \& Cox, R. W. (2013). Linear mixed-effects modeling approach to FMRI group analysis. NeuroImage, 1(73), 176-190. https://doi.org/10.1016/j.neuroimage. 2013.01.047

Cheng, D. T., Knight, D. C., Smith, C. N., \& Helmstetter, F. J. (2006). Human amygdala activity during the expression of fear responses. Behavioral neuroscience, 120(6), 1187. https://doi.org/10.1037/ 0735-7044.120.5.1187

Cheng, D. T., Knight, D. C., Smith, C. N., Stein, E. A., \& Helmstetter, F. J. (2003). Functional MRI of human amygdala activity during Pavlovian fear conditioning: stimulus processing versus response expression. Behavioral neuroscience, 117(1), 3. https://doi.org/10. 1037/0735-7044.117.1.3

Comte, M., Schön, D., Coull, J. T., Reynaud, E., Khalfa, S., Belzeaux, R., ... Weinberger, D. R. (2016). Dissociating bottom-up and top-down mechanisms in the cortico-limbic system during emotion processing. Cerebral Cortex, 26(1), 144-155.

Cox, R. W. (1996). AFNI: Software for analysis and visualization of functional magnetic resonance neuroimages. Computers and Biomedical Research, 29, 162-173.

Damasio, A. (1994). Descartes' error: Emotion, reason, and the human brain. New York, NY: Grosset/Putnam.

Dannlowski, U., Stuhrmann, A., Beutelmann, V., Zwanzger, P., Lenzen, T., Grotegerd, D., ... Bauer, J. (2012). Limbic scars: Long-term consequences of childhood maltreatment revealed by functional and structural magnetic resonance imaging. Biological Psychiatry, 71(4), 286-293.

Davidson, R. J. (2000). Affective style, psychopathology, and resilience: Brain mechanisms and plasticity. American Psychologist, 55(11), 1196.

Davidson, R. J. (2002). Anxiety and affective style: Role of prefrontal cortex and amygdala. Biological Psychiatry, 51(1), 68-80.

Davidson, R. J. (2003). Affective neuroscience and psychophysiology: Toward a synthesis. Psychophysiology, 40, 655-665.

De Bellis, M. D., Baum, A. S., Birmaher, B., Keshavan, M. S., Eccard, C. H., Boring, A. M., ... Ryan, N. D. (1999). Developmental traumatology part I: Biological stress systems. Biological psychiatry, 45(10), 1259-1270.

Dedovic, K., Duchesne, A., Engert, V., Lue, S. D., Andrews, J., Efanov, S. I., ... Pruessner, J. C. (2014). Psychological, endocrine and neural responses to social evaluation in subclinical depression. Social Cognitive and Affective Neuroscience, 9(10), 1632-1644. 
Dedovic, K., Renwick, R., Mahani, N. K., Engert, V., Lupien, S. J., \& Pruessner, J. C. (2005). The Montreal Imaging Stress Task: Using functional imaging to investigate the effects of perceiving and processing psychosocial stress in the human brain. Journal of Psychiatry Neuroscience, 30(5), 319-325.

Dedovic, K., Rexroth, M., Wolff, E., Duchesne, A., Scherling, C., Beaudry, T., ... Pruessner, J. C. (2009). Neural correlates of processing stressful information: An event-related fMRI study. Brain Research, 1293, 49-60.

Delgado, M. R., Nearing, K. I., LeDoux, J. E., \& Phelps, E. A. (2008). Neural circuitry underlying the regulation of conditioned fear and its relation to extinction. Neuron, 59(5), 829-838.

Diorio, D., Viau, V., \& Meaney, M. J. (1993). The role of the medial prefrontal cortex (cingulate gyrus) in the regulation of hypothalamic-pituitary-adrenal responses to stress. Journal of Neuroscience, 13(9), 3839-3847.

Dunn, E. C., Wang, Y., Tse, J., McLaughlin, K. A., Fitzmaurice, G., Gilman, S. E., \& Susser, E. S. (2017). Sensitive periods for the effect of childhood interpersonal violence on psychiatric disorder onset among adolescents. The British Journal of Psychiatry, 211(6), 365-372.

Eaton, D. K., Kann, L., Kinchen, S., Ross, J., Hawkins, J., Harris, W. A., \& Wechsler, H. (2006). Youth risk behavior surveillance-United States, 2005. Morbidity and Mortality Weekly Report, Surveillance Summaries, 55(SS05), 1-108. Retrieved from http://www.cdc.gov/ $\mathrm{mmwr} /$ preview/mmwrhtml/ss5505a1.htm

Fan, Y., Pestke, K., Feeser, M., Aust, S., Pruessner, J. C., Böker, H., ... Grimm, S. (2015). Amygdala-hippocampal connectivity changes during acute psychosocial stress: Joint effect of early life stress and oxytocin. Neuropsychopharmacology, 40(12), 2736.

Finkelhor, D., Ormrod, R. K., \& Turner, H. A. (2007). Poly-victimization: A neglected component in child victimization. Child Abuse \& Neglect, 31(1), 7-26.

Finkelhor, D., Ormrod, R. K., \& Turner, H. A. (2009). Lifetime assessment of poly-victimization in a national sample of children and youth. Child Abuse \& Neglect, 33(7), 403-411.

Finkelhor, D., Turner, T. A., Shattuck, A., \& Hamby, S. L. (2015). Prevalence of childhood exposure to violence, crime, and abuse results from the national survey of children's exposure to violence. The Journal of the American Medical Association Pediatrics, 169(8), 746-754. https://doi.org/10.1001/jamapediatrics.2015.0676

Forbes, D., Fletcher, S., Parslow, R., Phelps, A., O’Donnell, M., Bryant, R. A., ... Creamer, M. (2012). Trauma at the hands of another: Longitudinal study of differences in the posttraumatic stress disorder symptom profile following interpersonal compared with noninterpersonal trauma. The Journal of Clinical Psychiatry, 73(3), 372-376.

Gerin, M. I., Viding, E., Pingault, J. B., Puetz, V. B., Knodt, A. R., Radtke, S. R., ... McCrory, E. J. (2019). Heightened amygdala reactivity and increased stress generation predict internalizing symptoms in adults following childhood maltreatment. Journal of Child Psychology and Psychiatry, 60(7), 752-761.

Gilam, G., Maron-Katz, A., Kliper, E., Lin, T., Fruchter, E., Shamir, R., \& Hendler, T. (2017). Tracing the neural carryover effects of interpersonal anger on resting-state fMRI in men and their relation to traumatic stress symptoms in a subsample of soldiers. Frontiers in Behavioral Neuroscience, 11, 252.

Gilbertson, M. W., Shenton, M. E., Ciszewski, A., Kasai, K., Lasko, N. B., Orr, S. P., \& Pitman, R. K. (2002). Smaller hippocampal volume predicts pathologic vulnerability to psychological trauma. Nature Neuroscience, 5(11), 1242-1247. https://doi.org/10.1038/nn958

Gold, A. L., Morey, R. A., \& McCarthy, G. (2015). Amygdala-prefrontal cortex functional connectivity during threat-induced anxiety and goal distraction. Biological Psychiatry, 77(4), 394-403.
Goodman, A. M., Harnett, N. G., \& Knight, D. C. (2018). Pavlovian conditioned diminution of the neurobehavioral response to threat. Neuroscience and Biobehavioral Reviews, 84, 218-224.

Goodman, A. M., Wheelock, M. D., Harnett, N. G., Mrug, S., Granger, D. A., \& Knight, D. C. (2016). The hippocampal response to psychosocial stress varies with salivary uric acid level. Neuroscience, 339, 396-401. https://doi.org/10.1016/j.neuroscience.2016.10.002

Gross, J. J. (1998). The emerging field of emotion regulation: An integrative review. Review of General Psychology, 2(3), 271-299.

Hakamata, Y., Komi, S., Moriguchi, Y., Izawa, S., Motomura, Y., Sato, E., ... Inoue, Y. (2017). Amygdala-centred functional connectivity affects daily cortisol concentrations: A putative link with anxiety. Scientific Reports, 7(1), 1-11.

Hankin, B. L., \& Abramson, L. Y. (2001). Development of gender differences in depression: An elaborated cognitive vulnerabilitytransactional stress theory. Psychological Bulletin, 127(6), 773796. https://doi.org/10.1037/0033-2909.127.6.773

Hanson, R. F., Borntrager, C., Self-Brown, S., Kilpatrick, D. G., Saunders, B. E., Resnick, H. S., \& Amstadter, A. (2008). Relations among gender, violence exposure, and mental Health: The national survey of adolescents. American Journal of Orthopsychiatry, 78(3), 313-321. https://doi.org/10.1037/a0014056

Harding, K. A., \& Mezulis, A. (2017). Is rumination a risk and a protective factor? Europe's Journal of Psychology, 13(1), 28-46. https:// doi.org/10.5964/ejop.v13i1.1279

Hare, T. A., Camerer, C. F., \& Rangel, A. (2009). Self-control in decision-making involves modulation of the vmPFC valuation system. Science, 324(5927), 646-648.

Harnett, N. G., Wheelock, M. D., Wood, K. H., Goodman, A. M., Mrug, S., Elliott, M., ... Knight, D. C. (2019). Negative life experiences contribute to racial differences in the neural response to threat. NeuroImage, 202, 116086. https://doi.org/10.1016/j.neuroimage. 2019.116086

Hart, H., \& Rubia, K. (2012). Neuroimaging of child abuse: A critical review. Frontiers in Human Neuroscience, 6, 1-24.

Hayes, A. F. (2012). PROCESS: A versatile computational tool for observed variable mediation, moderation, and conditional process modeling [White paper]. Retrieved from http://www.afhayes.com/ public/process2012.pdf.

Hayes, A. F., \& Preacher, K. J. (2013). Conditional process modeling: Using structural equation modeling to examine contingent causal processes. In R. O. M. G. R. Hancock (Ed.), Structural equation modeling: A second course (2nd ed.). Charlotte, NC: Information Age Publishing.

Herringa, R. J., Birn, R. M., Ruttle, P. L., Burghy, C. A., Stodola, D. E., Davidson, R. J., \& Essex, M. J. (2013). Childhood maltreatment is associated with altered fear circuitry and increased internalizing symptoms by late adolescence. Proceedings of the National Academy of Sciences of the United States of America, 110(47), 19119-19124. https://doi.org/10.1073/pnas.1310766110

Hiser, J., \& Koenigs, M. (2018). The multifaceted role of the ventromedial prefrontal cortex in emotion, decision making, social cognition, and psychopathology. Biological Psychiatry, 83(8), 638-647.

Hooven, C., Nurius, P. S., Logan-Greene, P., \& Thompson, E. A. (2012). Childhood violence exposure: Cumulative and specific effects on adult mental health. Journal Of Family Violence, 27(6), 511-522.

Jiang, T., Webster, J. L., Robinson, A., Kassam-Adams, N., \& Richmond, T. S. (2018). Emotional responses to unintentional and intentional traumatic injuries among urban black men: A qualitative study. Injury, 49(5), 983-989.

Johnstone, T., van Reekum, C. M., Urry, H. L., Kalin, N. H., \& Davidson, R. J. (2007). Failure to regulate: Counterproductive recruitment of top-down prefrontal-subcortical circuitry in major depression. The Journal of Neuroscience, 27(33), 8877-8884. https://doi.org/10. 1523/JNEUROSCI.2063-07.2007 
Kalisch, R., Wiech, K., Critchley, H. D., \& Dolan, R. J. (2006). Levels of appraisal: A medial prefrontal role in high-level appraisal of emotional material. NeuroImage, 30(4), 1458-1466.

Karatsoreos, I. N., \& McEwen, B. S. (2011). Psychobiological allostasis: Resistance, resilience and vulnerability. Trends in Cognitive Sciences, 15(12), 576-584.

Kim, J. J., \& Diamond, D. M. (2002). The stressed hippocampus, synaptic plasticity and lost memories. Nature Reviews Neuroscience, 3(6), 453-462. https://doi.org/10.1038/nrn849

Klumpers, F., Kroes, M. C., Baas, J. M., \& Fernández, G. (2017). How human amygdala and bed nucleus of the stria terminalis may drive distinct defensive responses. Journal of Neuroscience, 37(40), 9645-9656.

Knight, D. C., Nguyen, H. T., \& Bandettini, P. A. (2005). The role of the human amygdala in the production of conditioned fear responses. NeuroImage, 26, 1193-1200. https://doi.org/10.1016/j.neuroimage. 2005.03.020

Knight, D. C., \& Wood, K. H. (2011). Investigating the neural mechanisms of aware and unaware fear memory with fMRI. Journal of Visualized Experiments, 56, e3083. https://doi.org/10.3791/3083

Lambert, H. K., Sheridan, M. A., Sambrook, K. A., Rosen, M. L., Askren, M. K., \& McLaughlin, K. A. (2017). Hippocampal contribution to context encoding across development is disrupted following early-life adversity. Journal of Neuroscience, 37(7), 1925-1934.

Lupien, S. J., McEwen, B. S., Gunnar, M. R., \& Heim, C. (2009). Effects of stress throughout the lifespan on the brain, behaviour and cognition. Nature Reviews Neuroscience, 10(6), 434-445. https://doi.org/ $10.1038 / \mathrm{nrn} 2639$

Margolin, G., \& Gordis, E. B. (2004). Children's exposure to violence in the family and community. Current Directions in Psychological Science, 13(4), 152-155.

Maron-Katz, A., Vaisvaser, S., Lin, T., Hendler, T., \& Shamir, R. (2016). A large-scale perspective on stress-induced alterations in restingstate networks [Article]. Scientific Reports, 6, 21503. https://doi. org $/ 10.1038 /$ srep21503

Mead, H. K., Beauchaine, T. P., \& Shannon, K. E. (2010). Neurobiological adaptations to violence across development. Developmental Psychopathology, 22(1), 1-37.

Menon, V. (2015). Salience network. In A. W. Toga (Ed.), Brain mapping: An encyclopedic reference (Vol. 2, pp. 597-611). Cambridge, MA: Academic Press.

Meulders, A., Meulders, M., \& Vlaeyen, J. W. S. (2014). Positive affect protects against deficient safety learning during extinction of fear of movement-related pain in healthy individuals scoring relatively high on trait anxiety. The Journal of Pain, 15(6), 632-644.

Moffitt, T. E. (2013). Childhood exposure to violence and lifelong health: Clinical intervention science and stress-biology research join forces. Development and Psychopathology, 25(4 0 2), 1619-1634. https:// doi.org/10.1017/S0954579413000801

Morawetz, C., Bode, S., Baudewig, J., \& Heekeren, H. R. (2017). Effective amygdala-prefrontal connectivity predicts individual differences in successful emotion regulation. Social cognitive and affective neuroscience, 12(4), 569-585.

Morey, R. A., Gold, A. L., LaBar, K. S., Beall, S. K., Brown, V. M., Haswell, C. C., ... McCarthy, G. (2012). Amygdala volume changes with posttraumatic stress disorder in a large case-controlled veteran group. Archives of General Psychiatry, 69(11), 1169-1178. https:// doi.org/10.1001/archgenpsychiatry.2012.50

Motzkin, J. C., Philippi, C. L., Wolf, R. C., Baskaya, M. K., \& Koenigs, M. (2015). Ventromedial prefrontal cortex is critical for the regulation of amygdala activity in humans. Biological psychiatry, 77(3), 276-284.

Mrug, S., Loosier, P. S., \& Windle, M. (2008). Violence exposure across multiple contexts: Individual and joint effects on adjustment. American Journal of Orthopsychiatry, 78(1), 70-84. https://doi. org/10.1037/0002-9432.78.1.70
Mrug, S., \& Windle, M. (2010). Prospective effects of violence exposure across multiple contexts on early adolescents' internalizing and externalizing problems. Journal of Child Psychology and Psychiatry, 51(8), 1-16. https://doi.org/10.1111/j.1469-7610.2010.02222.x

Mueller, S. C., Aouidad, A., Gorodetsky, E., Goldman, D., Pine, D. S., \& Ernst, M. (2013). Gray matter volume in adolescent anxiety: An impact of the brain-derived neurotrophic factor Val66Met polymorphism? Journal of the American Academy of Child \& Adolescent Psychiatry, 52(2), 184-195.

Nagai, Y., Critchley, H. D., Featherstone, E., Trimble, M. R., \& Dolan, R. J. (2004). Activity in ventromedial prefrontal cortex covaries with sympathetic skin conductance level: A physiological account of a "default mode" of brain function. NeuroImage, 22(1), 243-251.

Neumeister, P., Feldker, K., Heitmann, C., Buff, C., Brinkmann, L., Bruchmann, M., \& Straube, T. (2018). Specific amygdala response to masked fearful faces in post-traumatic stress relative to other anxiety disorders. Psychological Medicine, 48(7), 1209-1217.

Ochsner, K. N., Ray, R. R., Hughes, B., McRae, K., Cooper, J. C., Weber, J., ... Gross, J. J. (2009). Bottom-up and top-down processes in emotion generation: Common and distinct neural mechanisms. Psychological science, 20(11), 1322-1331.

Ochsner, K. N., Silvers, J. A., \& Buhle, J. T. (2012). Functional imaging studies of emotion regulation: A synthetic review and evolving model of the cognitive control of emotion. Annals of the New York Academy of Sciences 1251, E1-E24. https://doi.org/10.1111/j.17496632.2012.06751.x

Orem, T. R., Wheelock, M. D., Goodman, A. M., Harnett, N. G., Wood, K. H., Gossett, E. W., ... Knight, D. C. (2019). Amygdala and prefrontal cortex activity varies with individual differences in the emotional response to psychosocial stress. Behavioral Neuroscience, 133(2), 203.

Paret, C., Kluetsch, R., Zaehringer, J., Ruf, M., Demirakca, T., Bohus, M., ... Schmahl, C. (2016). Alterations of amygdala-prefrontal connectivity with real-time fMRI neurofeedback in BPD patients. Social Cognitive and Affective Neuroscience, 11(6), 952-960.

Phan, K. L., Fitzgerald, D. A., Nathan, P. J., Moore, G. J., Uhde, T. W., \& Tancer, M. E. (2005). Neural substrates for voluntary suppression of negative affect: A functional magnetic resonance imaging study. Biological Psychiatry, 57(3), 210-219.

Phelps, E. A. (2004). Human emotion and memory: interactions of the amygdala and hippocampal complex. Current Opinion in Neurobiology, 14, 198-202. https://doi.org/10.1016/j.conb.2004. 03.015

Phelps, E. A., Delgado, M. R., Nearing, K. I., \& LeDoux, J. E. (2004). Extinction learning in humans: Role of the amygdala and vmPFC. Neuron, 43(6), 897-905.

Protopopescu, X., Pan, H., Tuescher, O., Cloitre, M., Goldstein, M., Engelien, W., ... LeDoux, J. (2005). Differential time courses and specificity of amygdala activity in posttraumatic stress disorder subjects and normal control subjects. Biological psychiatry, 57(5), 464473.

Pruessner, J. C., Dedovic, K., Khalili-Mahani, N., Engert, V., Pruessner, M., Buss, C., ... Lupien, S. (2008). Deactivation of the limbic system during acute psychosocial stress: Evidence from positron emission tomography and functional magnetic resonance imaging studies. Biological Psychiatry, 63(2), 234-240.

Quaedflieg, C. W. E. M., Van De Ven, V., Meyer, T., Siep, N., Merckelbach, H. L. G. J., \& Smeets, T. (2015). Temporal dynamics of stress-induced alternations of intrinsic amygdala connectivity and neuroendocrine levels. PLOS ONE, 10(5), e0124141.

Quirk, G. J., \& Beer, J. S. (2006). Prefrontal involvement in the regulation of emotion: Convergence of rat and human studies. Current Opinion in Neurobiology, 16(6), 723-727.

Rauch, S. L., Delgado, M. R., Nearing, K. I., \& LeDoux, E. A. (2006). Neurocircuitry models of posttraumatic stress disorder and 
extinction: Human neuroimaging research - past, present, and future. Biological psychiatry, 60(4), 376-382.

Redlich, R., Opel, N., Bürger, C., Dohm, K., Grotegerd, D., Förster, K., ... Enneking, V. (2018). The limbic system in youth depression: Brain structural and functional alterations in adolescent in-patients with severe depression. Neuropsychopharmacology, 43(3), 546554.

Richter-Levin, G., \& Akirav, I. (2001). Amygdala-hippocampus dynamic interaction in relation to memory. Molecular Neurobiology, 22, 11-20.

Rorden, C., \& Brett, M. (2000). Stereotaxic display of brain lesions. Behavioural Neurology, 12(4), 191-200.

Roy, M., Shohamy, D., \& Wager, T. D. (2012). Ventromedial prefrontalsubcortical systems and the generation of affective meaning. Trends in Cognitive Sciences, 16(3), 147-156. https://doi.org/10.1016/j. tics.2012.01.005

Saltzman, K. M., Holden, G. W., \& Holahan, C. J. (2005). The psychobiology of children exposed to marital violence. Journal of Clinical Child and Adolescent Psychology, 34(1), 129-139.

Saxbe, D., Khoddam, H., Piero, L. D., Stoycos, S. A., Gimbel, S. I., Margolin, G., \& Kaplan, J. T. (2018). Community violence exposure in early adolescence: Longitudinal associations with hippocampal and amygdala volume and resting state connectivity. Developmental Science, 21(6), e12686.

Schuster, M. A., Elliott, M. N., Kanouse, D. E., Wallander, J. L., Tortolero, S. R., Ratner, J. A., ... Banspach, S. W. (2012). Racial and ethnic health disparities among fifth-graders in three cities. New England Journal of Medicine, 367(8), 735-745.

Schwab-Stone, M. E., Ayers, T. S., Kasprow, W., Voyce, C., Barone, C., Shriver, T., \& Weissberg, R. P. (1995). No safe haven: A study of violence exposure in an urban community. Journal of the American Academy of Child \& Adolescent Psychiatry, 34(10), 1343-1352.

Shin, L. M., Rauch, S. L., \& Pitman, R. K. (2006). Amygdala, medial prefrontal cortex, and hippocampal function in PTSD. Annals of the New York Academy of Sciences, 1071(1), 67-79.

Sinha, R., Lacadie, C. M., Constable, R. T., \& Seo, D. (2016). Dynamic neural activity during stress signals resilient coping. Proceedings of the National Academy of Sciences of the United States of America, 113(31), 8837-8842. https://doi.org/10.1073/pnas.1600965113

Smith, S. M., Jenkinson M., Woolrich, M. W., Beckmann, C. F., Behrens, T. E., Johansen-Berg, H., ... Matthews, P. M. (2004). Advances in functional and structural MR image analysis and implementation as FSL. NeuroImage, 23(1), S208-S219.

Sylvester, C. M., Corbetta, M., Raichle, M. E., Rodebaugh, T. L., Schlaggar, B. L., Sheline, Y. I., ... Lenze, E. J. (2012). Functional network dysfunction in anxiety and anxiety disorders. Trends in Neurosciences, 35(9), 527-535. https://doi.org/10.1016/j.tins.2012. 04.012

Taber-Thomas, B., \& Pérez-Edgar, K. (2015). Emerging adulthood brain development. In J. J. Arnett (Ed.), The Oxford handbook of emerging adulthood (pp. 126-141). Oxford, England: Oxford University Press. https://doi.org/10.1093/oxfordhb/9780199795574.013.15

Talairach, J., \& Tournoux, P. (1988). Co-planar stereotaxic atlas of the human brain. Leipzig, Germany: Theime.

Thayer, J. F., \& Lane, R. D. (2000). A model of neurovisceral integration in emotion regulation and dysregulation. Journal of Affective Disorders, 61(3), 201-216.

Thomason, M. E., \& Marusak, H. A. (2017). Toward understanding the impact of trauma on the early developing human brain. Neuroscience, 342, 55-67. https://doi.org/10.1016/j.neuroscience. 2016.02.022

Thomason, M. E., Marusak, H. A., Tocco, M. A., Vila, A. M., McGarragle, O., \& Rosenberg, D. R. (2015). Altered amygdala connectivity in urban youth exposed to trauma. Social Cognitive and Affective Neuroscience, 10(11), 1460-1468.
Turner, H. A., Finkelhor, D., \& Ormrod, R. (2010). Poly-victimization in a national sample of children and youth. American Journal of Preventive Medicine, 38(3), 323-330.

Turner, H. A., Shattuck, A., Finkelhor, D., \& Hamby, S. (2016). Polyvictimization and youth violence exposure across contexts. Journal of Adolescent Health, 58(2), 208-214.

van den Bulk, B. G., Somerville, L. H., van Hoof, M.-J., van Lang, N. D. J., van der Wee, N. J. A., Crone, E. A., \& Vermeiren, R. R. J. M. (2016). Amygdala habituation to emotional faces in adolescents with internalizing disorders, adolescents with childhood sexual abuse related PTSD and healthy adolescents. Developmental Cognitive Neuroscience, 21, 15-25.

van der Werff, S. J. A., Pannekoek, J. N., Veer, I. M., van Tol, M. J., Aleman, A., Veltman, D. J., ... van der Wee, N. J. A. (2013). Resting-state functional connectivity in adults with childhood emotional maltreatment. Psychological Medicine, 43, 1825-1836. https://doi.org/10.1017/S0033291712002942

van Harmelen, A.-L., van Tol, M.-J., Demenescu, L. R., van der Wee, N. J. A., Veltman, D. J., ... Elzinga, B. M. (2013). Enhanced amygdala reactivity to emotional faces in adults reporting childhood emotional maltreatment. Social Cognitive and Affective Neuroscience, 8(4), $362-369$.

Veer, I. M., Oei, N. Y. L., Spinhoven, P., van Buchem, M. A., Elzinga, B. M., \& Rombouts, S. A. R. B. (2011). Beyond acute social stress: Increased functional connectivity between amygdala and cortical midline structures. Neuroimage, 57(1534-1541). https://doi.org/ 10.1016/j.neuroimage.2011.05.074

Vyas, A., Mitra, R., Rao, B. S., \& Chattarji, S. (2002). Chronic stress induces contrasting patterns of dendritic remodeling in hippocampal and amygdaloid neurons. Journal of Neuroscience, 22(15), 6810 6818

Wang, L., Dai, Z., Peng, H., Tan, L., Ding, Y., He, Z., Zhang, Y. ... Li, W. (2014). Overlapping and segregated resting-state functional connectivity in patients with major depressive disorder with and without childhood neglect. Human Brain Mapping, 35(4), 1154-1166.

Watson, D., Clark, L. A., \& Tellegen, A. (1988). Development and validation of brief measures of positive and negative affect: The PANAS scales. Journal of Personality and Social Psychology, 54(6), 1063-1070.

Weissman, D. G., Jenness, J. L., Colich, N. L., Miller, A. B., Sambrook, K. A., Sheridan, M. A., \& McLaughlin, K. A. (2019). Altered neural processing of threat-related information in children and adolescents exposed to violence: A transdiagnostic mechanism contributing to the emergence of psychopathology. Journal of the American Academy of Child \& Adolescent Psychiatry(19), 1-11. https://doi. org/10.1016/j.jaac.2019.08.471

Weissman, D. G., Lambert, H. K., Rodman, A. M., Peverill, M., Sheridan, M. A., \& McLaughlin, K. A. (2020). Reduced hippocampal and amygdala volumes as mechanisms of stress sensitization to depression following childhood violence exposure. Depression and Anxiety, 1-10. https://doi.org/10.1002/da.23062

Wheelock, M. D., Harnett, N. G., Wood, K. H., Orem, T. R., Granger, D. A., Mrug, S., \& Knight, D. C. (2016). Prefrontal cortex activity is associated with the biobehavioral components of the stress response. Frontiers in Human Neuroscience, 10, 1-12. https://doi.org/10. 3389/fnhum.2016.00583

Wheelock, M. D., Rangaprakash, D., Harnett, N. G., Wood, K. H., Orem, T. R., Mrug, S., ... Knight, D. C. (2018). Psychosocial stress reactivity is associated with decreased whole-brain network efficiency and increased amygdala centrality. Behavioral Neuroscience, 132(6), 561-572. https://doi.org/10.1037/bne0000276

White, S. F., Costanzo, M. E., Blair, J. R., \& Roy, M. J. (2015). PTSD symptom severity is associated with increased recruitment of topdown attentional control in a trauma-exposed sample. NeuroImage: Clinical, 7, 19-27. 
Windle, M., Grunbaum, J., Elliott, M., Tortolero, S. R., Berry, S., Gilliland, J., ... Schuster, M. (2004). Healthy passages: A multilevel, multimethod longitudinal study of adolescent health. American Journal of Preventive Medicine, 27(2), 164-172. https://doi.org/10. 1016/j.amepre.2004.04.007

Wood, K. H., Ver Hoef, L. W., \& Knight, D. C. (2014). The amygdala mediates the emotional modulation of threat-elicited skin conductance response. Emotion, 14(4), 693. https://doi.org/10.1037/ a0036636

Wood, K. H., Wheelock, M. D., Shumen, J. R., Bowen, K. H., Ver Hoef, L. W., \& Knight, D. C. (2015). Controllability modulates the neural response to predictable but not unpredictable threat in humans. NeuroImage, 119, 371-381. https://doi.org/10.1016/j.neuroimage. 2015.06.086
Yamamoto, T., Toki, S., Siegle, G. J., Takamura, M., Takaishi, Y., Yoshimura, S., ... Yamawaki, S. (2017). Increased amygdala reactivity following early life stress: A potential resilience enhancer role. BMC Psychiatry, 17(27). https://doi.org/10.1186/s12888-017-1201-

Young, L., \& Koenigs, M. (2007). Investigating emotion in moral cognition: A review of evidence from functional neuroimaging and neuropsychology. British Medical Bulletin, 84, 69-79. https://doi. org/10.1093/bmb/ldm031

Publisher's note Springer Nature remains neutral with regard to jurisdictional claims in published maps and institutional affiliations. 